\title{
Enhancement of Fluorescent Labeling via a Composited Thin Film
}

\author{
Taikei Suyama, ${ }^{1}$ Xiaowei Ji, ${ }^{2}$ and Yaoju Zhang ${ }^{3}$ \\ ${ }^{1}$ Department of Electrical and Computer Engineering, Akashi National College of Technology, Akashi 674-8501, Japan \\ ${ }^{2}$ Graduate School of Science and Technology, Kumamoto University, Kumamoto 860-8555, Japan \\ ${ }^{3}$ College of Physics and Electronic Information Engineering, Wenzhou University, Wenzhou 325035, China
}

Correspondence should be addressed to Taikei Suyama; suyama@akashi.ac.jp

Received 2 August 2013; Accepted 30 January 2014; Published 3 March 2014

Academic Editor: Nanda Gopal Sahoo

Copyright (C) 2014 Taikei Suyama et al. This is an open access article distributed under the Creative Commons Attribution License, which permits unrestricted use, distribution, and reproduction in any medium, provided the original work is properly cited.

\begin{abstract}
Fluorescent labeling is the prevailing imaging technique in cell biological research. When statistical investigations on a large number of cells are involved, experimental study is required for both low magnification to get a reliable statistical population and high contrast to achieve accurate diagnosis on the nature of the cells' perturbation. As microscope objectives of low magnification generally yield low collection efficiency, such studies are limited by the fluorescence signal weakness. To overcome this technological insufficiency, Le Moal et al. proposed a method based on metal-coated substrates that enhanced the fluorescence process and improved collection efficiency in fluorescence microscope observation and that could be directly used with a common microscope setup. In this paper, we use an $\mathrm{Ag}-\mathrm{Si}_{3} \mathrm{~N}_{4}-\mathrm{Ag}$ multilayer film coated on the substrate and numerically analyse the optical behavior of a fluorophore which was placed above the composited film coated on the substrate. The results shows that by using an $\mathrm{Ag}-\mathrm{Si}_{3} \mathrm{~N}_{4}-\mathrm{Ag}$ composited film the fluorescence imaging can be enhanced remarkably.
\end{abstract}

\section{Introduction}

Many biological studies and applications involve biomolecule detection by means of fluorescence. The use of fluorescent markers is notably the most widespread labeling technique for cell imaging. A large range of fluorophores with distinctive spectral characteristics is available, providing many possibilities for multicolor biological labeling. Using a multicolor set of functionalized dyes (biomarkers) and appropriate optical filters, one can selectively image parts of the cell or track several active biomolecules inside the cell. However, the extinction coefficient of these fluorescent molecules is relatively weak compared with those of fluorescent nanoparticles. Consequently, the detection of low concentrations of fluorophores is limited by the fluorescence signal weakness. Another drawback of molecular fluorophores is their low photostability. When an assembly of fluorophores is continuously excited, their emitted light will progressively fade as light induces their destruction. Signal intensities can then hardly be standardized.
Signal weakness and photoreactivity have been partly overcome as a result of recent progress in the use of semiconductor nanocrystals, commonly called quantum dots, in biology. However, they introduce new problems such as blinking of the emission signal and low biocompatibility that restrict the scope of in vivo applications.

Despite the increasing interest raised by fluorescent nanoparticles, the use of fluorescent molecules as markers for biomedical applications still has relevance and attractiveness. In addition to the development of new fluorescent markers, the renewed interest in standard molecular fluorophores had been motivated by the development of innovative optical techniques for fluorescence enhancement.

In recent years, metal surfaces play a unique role in many biomolecular techniques such as the self-assembly and selforganization of proteins and lipids [1-3]. A thin metal film can produce the surface plasmon-coupled emission (SPCE). The surface plasmon-coupled emission method was proposed as a high sensitivity and efficient fluorescence detection method by Lakowicz et al. $[4,5]$. They showed, through 


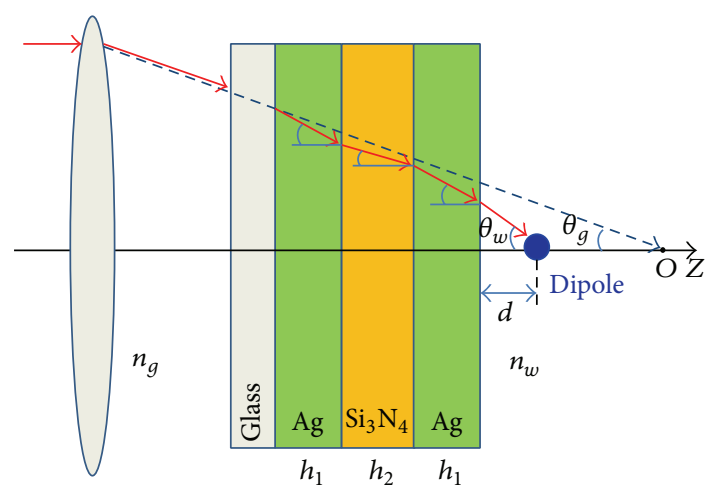

FIGURE 1: Focusing of excitation light passing through the multilayer films.

imaging fluorophores near to a silver-coated glass substrate, that the fluorescence emission was highly directional and this has increased the collection efficiency to nearly $50 \%$ as well as contributed to low background noise. SPCE has found a number of applications in areas such as biotechnology and biological measurements $[6,7]$.
In this work, we consider fluorophores to be placed above an $\mathrm{Ag}-\mathrm{Si}_{3} \mathrm{~N}_{4}$-Ag multilayer film surface and describe the physical mechanisms of fluorescence enhancement. The optical behavior of the fluorophores was simulated by implementing a simple electric-dipole model.

\section{Theory}

As shown in Figure 1, we assume that the oil filled in between the objective lens and the cover glass has the same refractive index with the cover glass; a metal-dielectric multilayer film is coated on the cover glass. Fluorescent molecule is located near the focal point of the objective lens at a distance $d$ from the metal-dielectric multilayer film surface. In order to efficiently excite the fluorescence, in this paper, we consider a radially polarized beam incident on the objective lens.

2.1. Excitation of Dipole in the Object Space. Using the method proposed by Török et al. [8, 9], the excitation field $E_{w}$ in the object space is simply given by

$$
E_{w}=\left[\begin{array}{l}
E_{w, r} \\
E_{w, \varphi} \\
E_{w, z}
\end{array}\right]=\left[\begin{array}{c}
i \int_{0}^{\theta_{g, \max }} t_{p} \cos \theta_{w} \sqrt{\cos \theta_{g}} \sin \theta_{g} J_{1}\left(k_{g} r \sin \theta_{g}\right) \exp (i \Phi) \exp \left[i k_{w} z \cos \theta_{w}\right] d \theta_{g} \\
0 \\
-i \int_{0}^{\theta_{g, \max }} t_{p} \sin \theta_{w} \sqrt{\cos \theta_{g}} \sin \theta_{g} J_{0}\left(k_{g} r \sin \theta_{g}\right) \exp (i \Phi) \exp \left[i k_{w} z \cos \theta_{w}\right] d \theta_{g}
\end{array}\right],
$$

where $\theta_{g, \max }$ is the focusing angle of objective lens. Obviously, as the dipole is excited by a $p$-polarized incident plane wave, the electric field component in the direction of the polar angle is zero.
2.2. Electric Field in the Image Space. With the vector diffraction theory and some methods that are proposed by Richards et al. [10-14], we can obtain the expression of the electric field $E_{a}$ in the image space. It is simply given by

$$
E_{a}(r, \varphi, z)=\left[\begin{array}{c}
E_{a, r} \\
E_{a, \varphi} \\
E_{a, z}
\end{array}\right]=\left[\begin{array}{c}
-\frac{i k_{a}}{2}\left\{\mu \sin \theta_{d} \cos \left(\phi_{d}-\varphi\right)\left[K_{0}^{\mathrm{I}}+K_{2}^{\mathrm{I}}\right]-\mu \cos \theta_{d}\left[2 i K_{1}^{\mathrm{I}}\right]\right\} \\
-\frac{i k_{a}}{2}\left\{\mu \sin \theta_{d} \sin \left(\phi_{d}-\varphi\right)\left[K_{0}^{\mathrm{I}}-K_{2}^{\mathrm{I}}\right]\right\} \\
-\frac{i k_{a}}{2}\left\{\mu \sin \theta_{d} \sin \left(\phi_{d}-\varphi\right)\left[K_{0}^{\mathrm{I}}-K_{2}^{\mathrm{I}}\right]-\mu \cos \theta_{d}\left[2 i K_{0}^{\Pi}\right]\right\}
\end{array}\right]
$$

where

$$
\begin{aligned}
K_{0}^{\mathrm{I}}=\int_{0}^{\sigma} & \sqrt{\frac{\cos \theta_{a}}{\cos \theta_{g}}} \sin \theta_{a}\left(\tau_{s}+\tau_{p} \cos \theta_{w} \cos \theta_{a}\right) \\
& \times J_{0}\left(k_{a} r \sin \theta_{a}\right) \exp \left(i k_{a} z \cos \theta_{a}\right) \exp (i \Phi) d \theta_{a} \\
K_{1}^{\mathrm{I}=} \int_{0}^{\sigma} & \sqrt{\frac{\cos \theta_{a}}{\cos \theta_{g}}} \sin \theta_{a} \times \tau_{p} \sin \theta_{w} \cos \theta_{a} \\
& \times J_{1}\left(k_{a} r \sin \theta_{a}\right) \exp \left(i k_{a} z \cos \theta_{a}\right) \exp (i \Phi) d \theta_{a} \\
K_{2}^{\mathrm{I}}= & \int_{0}^{\sigma} \sqrt{\frac{\cos \theta_{a}}{\cos \theta_{g}}} \sin \theta_{a}\left(\tau_{s}-\tau_{p} \cos \theta_{w} \cos \theta_{a}\right) \\
& \times J_{2}\left(k_{a} r \sin \theta_{a}\right) \exp \left(i k_{a} z \cos \theta_{a}\right) \exp (i \Phi) d \theta_{a}
\end{aligned}
$$

$$
\begin{aligned}
K_{0}^{\Pi=} \int_{0}^{\sigma} & \sqrt{\frac{\cos \theta_{a}}{\cos \theta_{g}}} \sin \theta_{a} \times \tau_{p} \sin \theta_{w} \sin \theta_{a} \\
& \times J_{0}\left(k_{a} r \sin \theta_{a}\right) \exp \left(i k_{a} z \cos \theta_{a}\right) \exp (i \Phi) d \theta_{a} \\
K_{1}^{\Pi=} \int_{0}^{\sigma} & \sqrt{\frac{\cos \theta_{a}}{\cos \theta_{g}}} \sin \theta_{a} \times \tau_{p} \cos \theta_{w} \sin \theta_{a} \\
& \times J_{1}\left(k_{a} r \sin \theta_{a}\right) \exp \left(i k_{a} z \cos \theta_{a}\right) \exp (i \Phi) d \theta_{a},
\end{aligned}
$$

where $J_{n}(\cdot)$ is the Bessel function of the first kind of $n$ order, $\Phi$ is the wavefront aberration function, the subscript $w, a, g$ denote that the variable quantity is located in the objective 


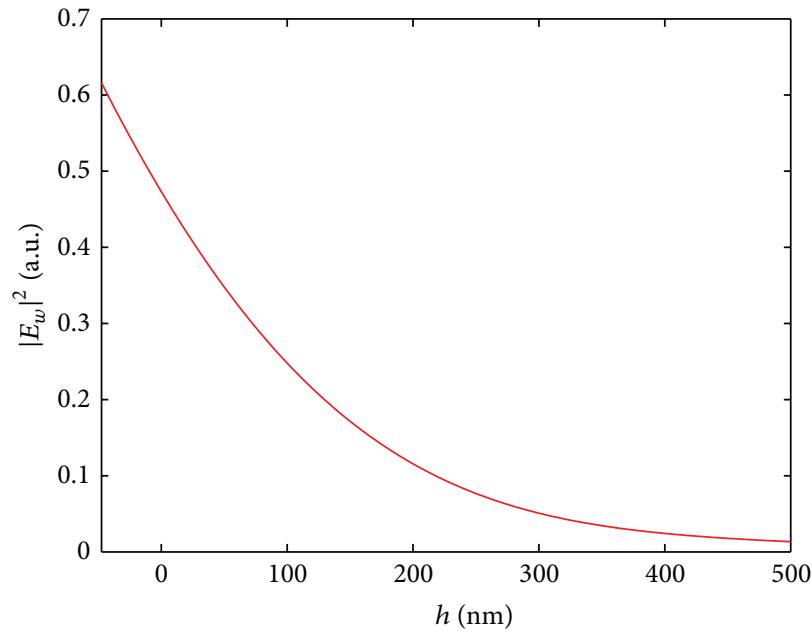

(a) The case of the $\mathrm{Ag}-\mathrm{Si}_{3} \mathrm{~N}_{4}-\mathrm{Ag}$ multilayer film

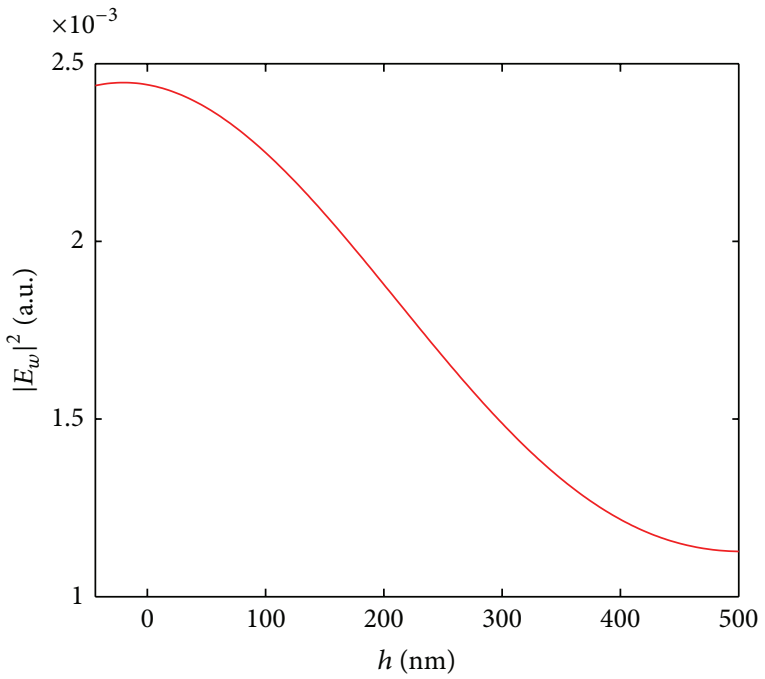

(b) The case of the single-layer metal film

FIGURE 2: Intensity at the electric dipole versus the distance $h$ between the interface and geometric focus.

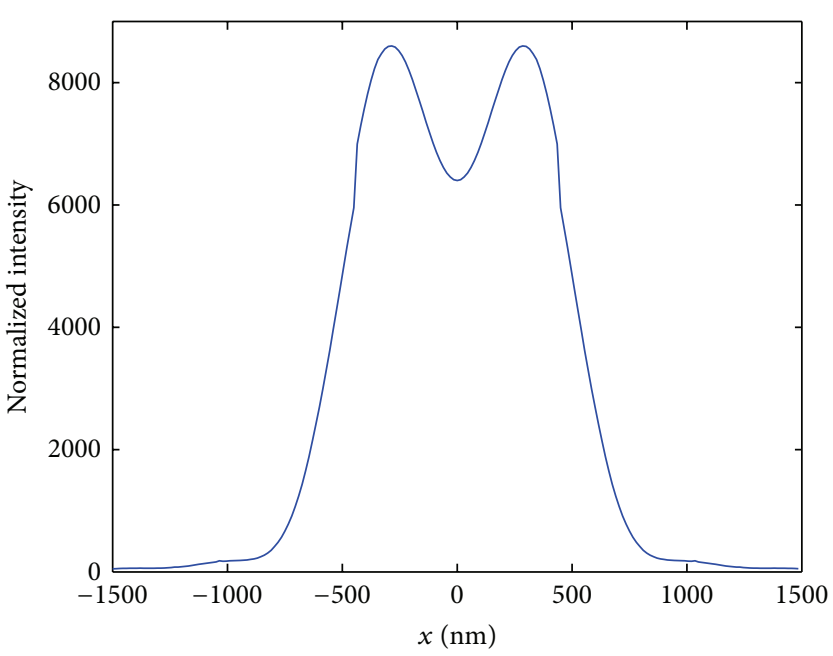

(a) The multilayer film with $h_{1}=5.25 \mathrm{~nm}$ and $h_{2}=37 \mathrm{~nm}$

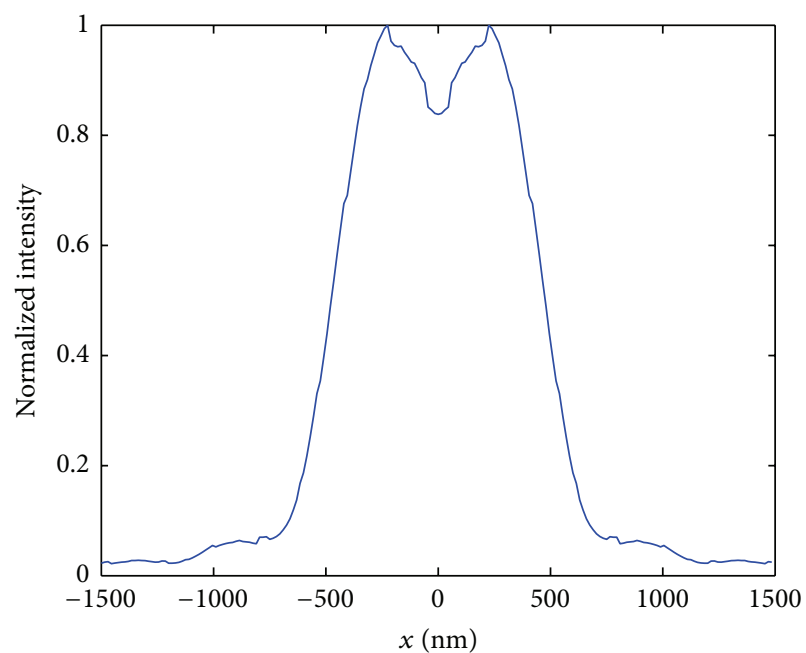

(b) The single-layer metal film with $h_{1}=44 \mathrm{~nm}$

FIGURE 3: Normalized intensity of emission by a dipole along the $x$ direction in the focal plane of $z=0$.

space, the imaging space, and the space between the objective lens and the cover glass, respectively.

2.3. Intensity in Image Space. The intensity that is detected in the image space is

$$
\frac{I \propto\left(\left|E_{a, r}\right|^{2}+\left|E_{a, \varphi}\right|^{2}+\left|E_{a, z}\right|^{2}\right)}{P_{T}},
$$

where the normalization factor $P_{T}$ is the total power emitted by the dipole in the presence of a metal interface $[15,16]$. For a dipole which is perpendicularly oriented to the metal interface, $\theta_{d}=0^{\circ}$, the intensity is rotationally symmetric.
Consider the following:

$$
\frac{I_{\perp} \propto 4\left(\left|K_{1}^{\mathrm{I}}\right|^{2}+\left|K_{0}^{\Pi}\right|^{2}\right)}{P_{T}} .
$$

On the other hand, the image of a dipole which has an orientation parallel to the metal interface along the $x$ direction is not rotationally symmetric; that is, it has a dependence on $\phi$.

$$
\frac{I_{\|} \propto\left(\left|K_{0}^{\mathrm{I}}\right|^{2}+\left|K_{2}^{\mathrm{I}}\right|^{2}+4 \cos ^{2} \varphi\left|K_{1}^{\Pi}\right|^{2}+2 \cos 2 \varphi \operatorname{Re}\left[K_{0}^{\mathrm{I}} K_{2}^{\mathrm{I}}\right]\right)}{P_{T}} .
$$



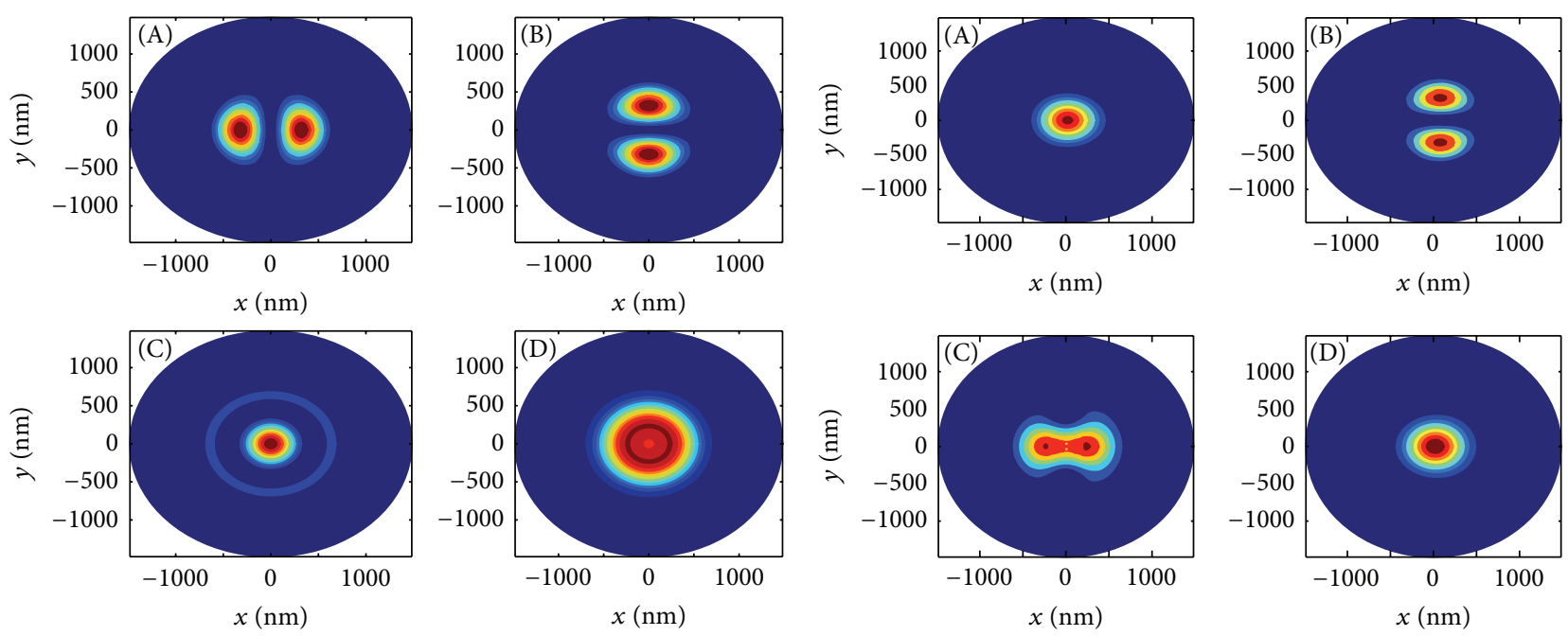

(a) $\theta_{d}=\varphi_{d}=0^{\circ}$
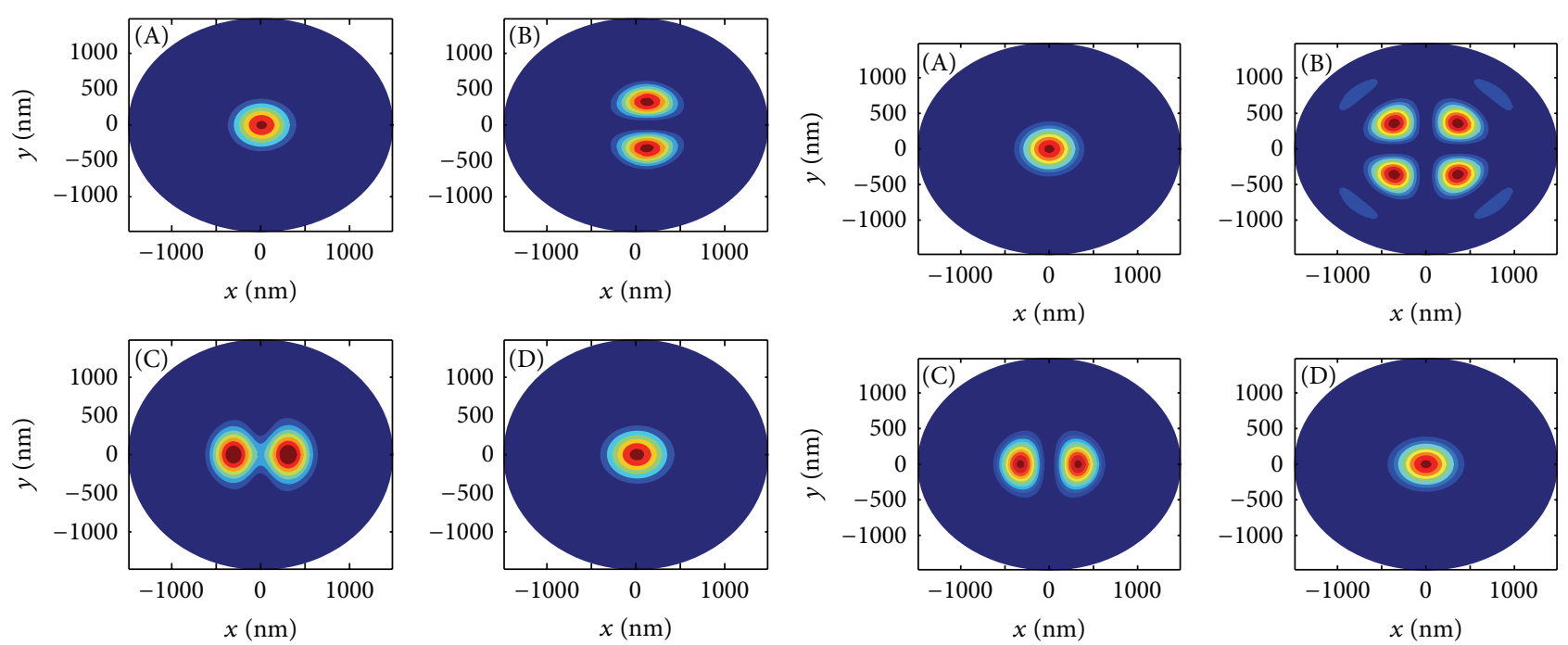

(c) $\theta_{d}=\frac{\pi}{3}, \varphi_{d}=0^{\circ}$
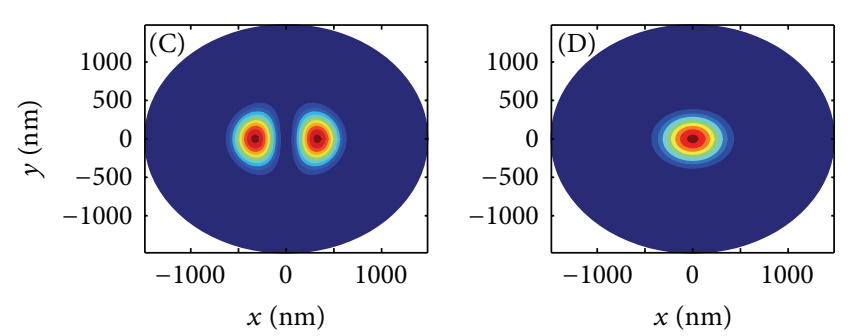

(d) $\theta_{d}=\frac{\pi}{2}, \varphi_{d}=0^{\circ}$

FIGURE 4: Intensity distribution of emission by a dipole with different orientation angles in the focal plane in image space. (A), (B), and (C) are the component intensities of $\left|E_{a x}\right|^{2},\left|E_{a y}\right|^{2}$, and $\left|E_{a z}\right|^{2}$ respectively; (D) is the total intensity of $\left|E_{a x}\right|^{2}+\left|E_{a y}\right|^{2}+\left|E_{a z}\right|^{2}$.

\section{Numerical Results}

We assume that the thickness of the metal film and the dielectric film are $h_{1}$ and $h_{2}$, respectively. $h$ is the distance between the interface and the geometric focus of the objective lens, and $\theta_{g, \max }=60^{\circ}, d=20 \mathrm{~nm}$. In this paper, we assume that the metallic material is silver $(\mathrm{Ag})$, which have different refractive indexes of exciting light and emitted light. Our numerical results showed that the larger the refractive index of the dielectric film between the metal films was, the more beneficial to strengthen the two-way transmissions of light it is. To this end, we take a high refractive index material of amorphous silicon $\left(\mathrm{Si}_{3} \mathrm{~N}_{4}\right)$. At first, we make a comparison of the maximal intensity of the focused light spot in two cases: one is using an $\mathrm{Ag}-\mathrm{Si}_{3} \mathrm{~N}_{4}$-Ag multilayer film coated on the cover glass, another is using a single-layer metal film. As shown in Figure 2, we can see that, in the first case of using the Ag$\mathrm{Si}_{3} \mathrm{~N}_{4}$-Ag multilayer film, when $h=-2 h_{1}-h_{2}=47.5 \mathrm{~nm}$, the intensity of focused light spot obtains the maximum value, $\left|E_{w}\right|_{\max }^{2}=0.616$. And in the case of using a single metal film, when $h_{1}=44 \mathrm{~nm}, h=-21 \mathrm{~nm}$, the intensity of the focused light spot obtains the maximum value, $\left|E_{w}\right|_{\max }^{2}=2.446 \times 10^{-3}$. Here the negative $h$ means that the right surface of the Ag$\mathrm{Si}_{3} \mathrm{~N}_{4}$-Ag multilayer film should be in the right side of the geometric focus of the objective lens, as shown in Figure 1. It is obvious that the intensity of the spot center can be enhanced by about 251 times using an $\mathrm{Ag}-\mathrm{Si}_{3} \mathrm{~N}_{4}-\mathrm{Ag}$ multilayer film coated on the cover glass than the single-layer metal film.

As shown as in Figure 3, we compared the normalized imaging intensity of emission by the dipole along the $x$ direction in the focal plane in the case of using two different films: an $\mathrm{Ag}-\mathrm{Si}_{3} \mathrm{~N}_{4}$-Ag multilayer film and a single-layer metal film. Orientation angles of the dipole are $\theta_{d}=\varphi_{d}=0^{\circ}$. Intensities are normalized to the maximum imaging intensity in case of using the single-layer metal film. The figure shows that, using 


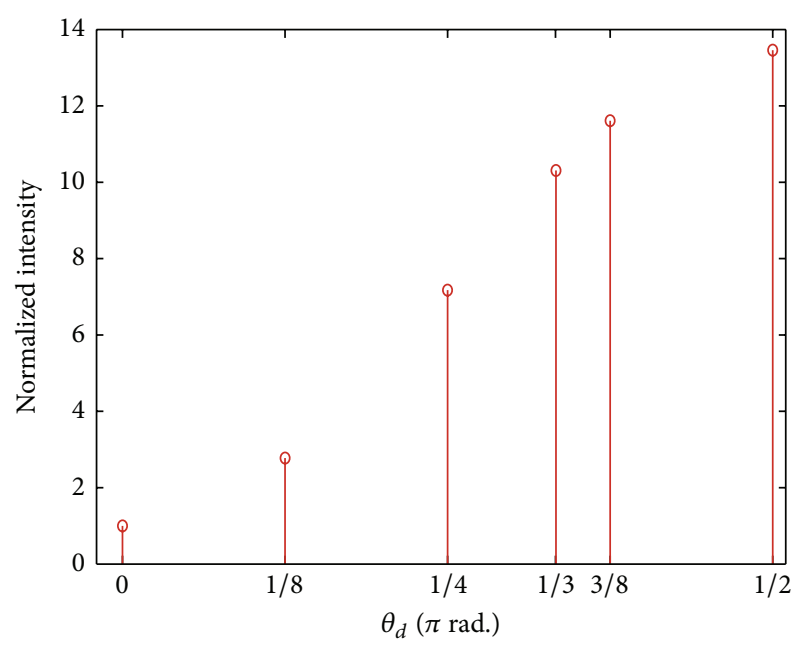

(a)

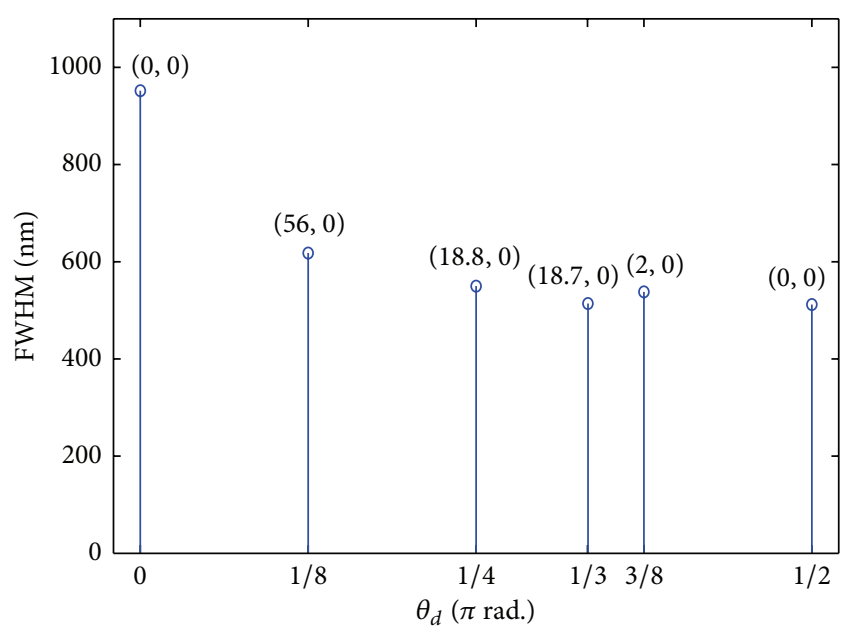

(b)

Figure 5: Central intensity (a) of spot and its FWHM (b) for the total intensity versus the pole angle of the dipole, where numbers atop each line denote the $(x, y)$ coordinates of the spot center.

the $\mathrm{Ag}-\mathrm{Si}_{3} \mathrm{~N}_{4}-\mathrm{Ag}$ multilayer film coated on the cover glass, the imaging intensity of the fluorescence can be enhanced by thousands of times than using a single-layer metal film. This may overcome the drawback of molecular fluorophores such as signal weakness and photoreactivity.

Then we study the correlation between imaging facula and orientation of a single dipole. The intensity distributions of emitted light by a dipole with different orientation angles on the focal plane in imaging space are shown in Figure 4. In these figures, we represented the intensity distributions of components $\left|E_{a x}\right|^{2},\left|E_{a y}\right|^{2},\left|E_{a z}\right|^{2}$ and the total intensity of $\left|E_{a x}\right|^{2}+\left|E_{a y}\right|^{2}+\left|E_{a z}\right|^{2}$ with different orientation angles. Comparing these charts of the intensity distribution, despite the imaging intensity distributions of the dipole with different orientations have a same look, the strength and the size of the light spot are different.

Finally, we study the light intensity of the total light intensity imaging facula center and the full width at half maximum versus the pole angle of dipole. As shown as in Figure 5, with the increase of dipole azimuth (electric dipole moment by parallel optical axis to the vertical axis), the intensity of the light spot is increased gradually. When the dipole moment is parallel to the optical axis, the size of the imaging facula is larger. When the dipole moment is perpendicular to the optical axis, the size of the imaging facula is smaller. If a polarizer is placed in front of the detector, the imaging of $x$ or $y$ polarization component can be obtained.

The intensity patterns $\left(\left|E_{a x}\right|^{2},\left|E_{a y}\right|^{2}\right)$ of $x$ and $y$ polarization component or the positions change with the azimuth $\theta_{d}$. These results indicate that, using a metal-dielectric multilayer film, a single dipole can be detected. And this can be used in many optical devices.

\section{Conclusion}

In this paper, we assumed that fluorophores are placed above an $\mathrm{Ag}-\mathrm{Si}_{3} \mathrm{~N}_{4}$ - $\mathrm{Ag}$ multilayer film surface and we have described the physical mechanisms of fluorescence enhancement. The optical behavior of the fluorophores was simulated by implementing a simple electric-dipole model and comparing the results with a single-layer metal film. The results shows that by using a metal-dielectric multilayer film, the intensity of the spot center can be enhanced by 251 times than using a thin single-layer metal film. Hence, the intensity of the fluorescence imaging can be increased by thousands of times. With the increase of dipole azimuth (electric dipole moment by parallel optical axis to the vertical axis), the intensity of the light spot is increased gradually. This may overcome the shortcomings of traditional fluorescence such as low imaging intensity and difficulty in detection.

\section{Conflict of Interests}

The authors declare that there is no conflict of interests regarding the publication of this paper.

\section{Acknowledgment}

This work was supported in part by Grants-in-Aid for Scientific Research from Japan Society for the Promotion of Science (Grant no. 23560404).

\section{References}

[1] E. Le Moal, E. Fort, S. Lévêque-Fort, F. P. Cordelières, M.-P. Fontaine-Aupart, and C. Ricolleau, "Enhanced fluorescence cell imaging with metal-coated slides," Biophysical Journal, vol. 92, no. 6, pp. 2150-2161, 2007.

[2] T. Suyama, Y. Okuno, and T. Matsuda, "Enhancement of TM-TE mode conversion caused by excitation of surface plasmons on a metal grating and its application for refractive index measurement," Progress in Electromagnetics Research, vol. 72, pp. 91-103, 2007.

[3] T. Suyama and Y. Zhang, "3D super-resolution fluorescence microscopy using cylindrical vector beams," Progress in Electromagnetics Research Letters, vol. 43, pp. 73-81, 2013. 
[4] J. R. Lakowicz, J. Malicka, I. Gryczynski, and Z. Gryczynski, "Directional surface plasmon-coupled emission: a new method for high sensitivity detection," Biochemical and Biophysical Research Communications, vol. 307, no. 3, pp. 435-439, 2003.

[5] J. R. Lakowicz, "Radiative decay engineering 3. Surface plasmon-coupled directional emission," Analytical Biochemistry, vol. 324, no. 2, pp. 153-169, 2004.

[6] F. D. Stefani, K. Vasilev, N. Bocchio, N. Stoyanova, and M. Kreiter, "Surface-plasmon-mediated single-molecule fluorescence through a thin metallic film," Physical Review Letters, vol. 94, no. 2, Article ID 023005, 2005.

[7] W. T. Tang, E. Chung, Y.-H. Kim, P. T. C. So, and C. J. R. Sheppard, "Investigation of the point spread function of surface plasmon-coupled emission microscopy," Optics Express, vol. 15, no. 8, pp. 4634-4646, 2007.

[8] P. Török, P. Varga, Z. Laczik, and G. R. Booker, "Electromagnetic diffraction of light focused through a planar interface between materials of mismatched refractive indices: structure of the electromagnetic field," Journal of the Optical Society of America A, vol. 12, no. 2, pp. 325-332, 1995.

[9] A. S. Van De Nes, L. Billy, S. F. Pereira, and J. J. M. Braat, "Calculation of the vectorial field distribution in a stratified focal region of a high numerical aperture imaging system," Optics Express, vol. 12, no. 7, pp. 1281-1293, 2004.

[10] B. Richards and E. Wolf, "Electromagnetic diffraction in optical systems. II. Structure of the image field in an aplanatic system," Proceedings of the Royal Society A, vol. 253, pp. 358-379, 1959.

[11] P. Török, P. D. Higdon, and T. Wilson, "Theory for confocal and conventional microscopes imaging small dielectric scatterers," Journal of Modern Optics, vol. 45, no. 8, pp. 1681-1698, 1998.

[12] P. Torok, P. Varga, Z. Laczik, and G. R. Booker, "Electromagnetic diffraction of light focused through a planar interface between materials of mismatched refractive indices: an integral representation," Journal of the Optical Society of America A, vol. 12, no. 2, pp. 325-332, 1995.

[13] P. D. Higdon, P. Török, and T. Wilson, "Imaging properties of high aperture multiphoton fluorescence scanning optical microscopes," Journal of Microscopy, vol. 193, no. 2, pp.127-141, 1999.

[14] H. F. Arnoldus and J. T. Foley, "Transmission of dipole radiation through interfaces and the phenomenon of anti-critical angles," Journal of the Optical Society of America A, vol. 21, no. 6, pp. 1109-1117, 2004.

[15] J. Enderlein and M. Böhmer, "Influence of interface-dipole interactions on the efficiency of fluorescence light collection near surfaces," Optics Letters, vol. 28, no. 11, pp. 941-943, 2003.

[16] E. H. Hellen and D. Axelrod, "Fluorescence emission at dielectric and metal-film interfaces," Journal of the Optical Society of America B, vol. 4, pp. 337-349, 1987. 

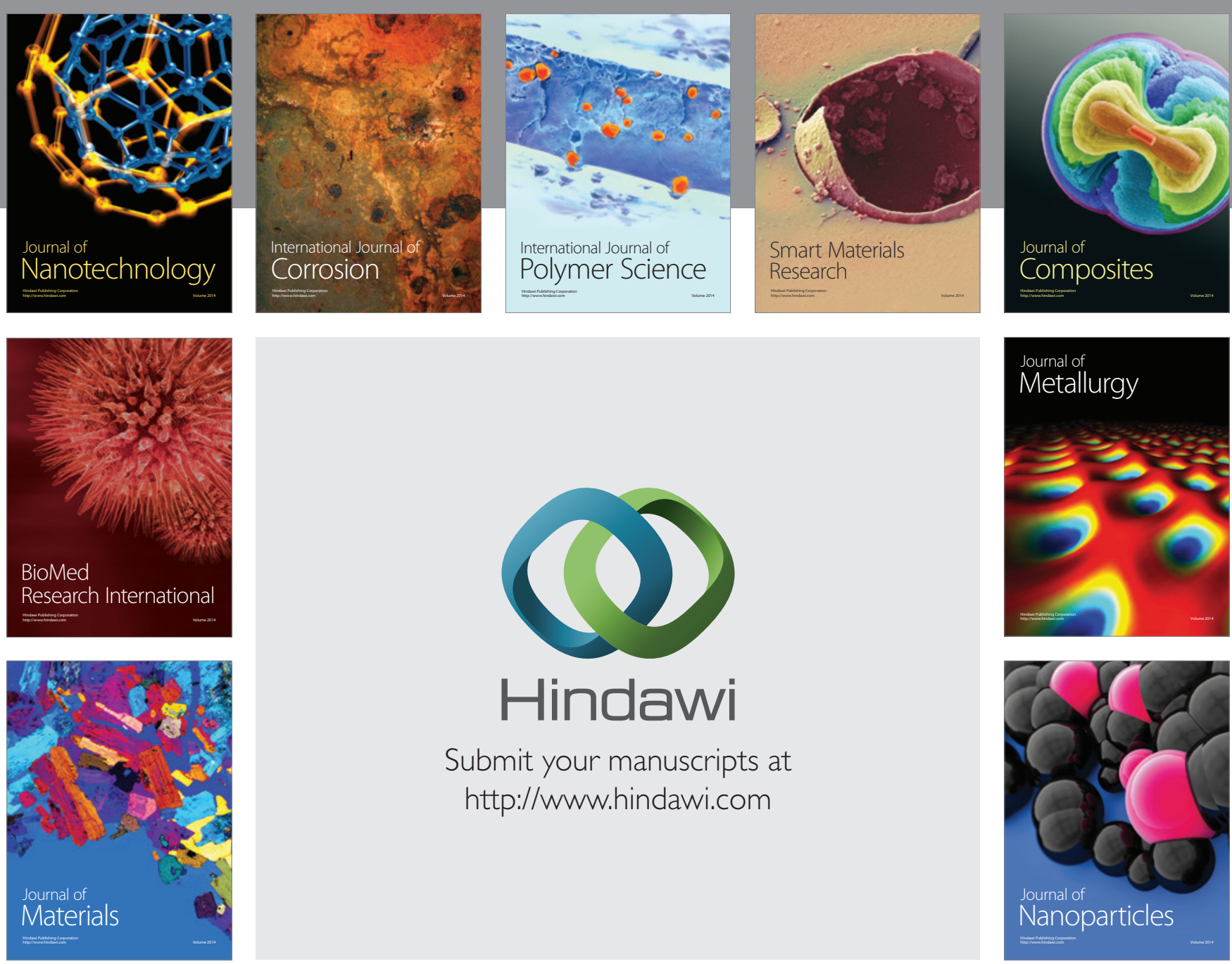

Submit your manuscripts at http://www.hindawi.com
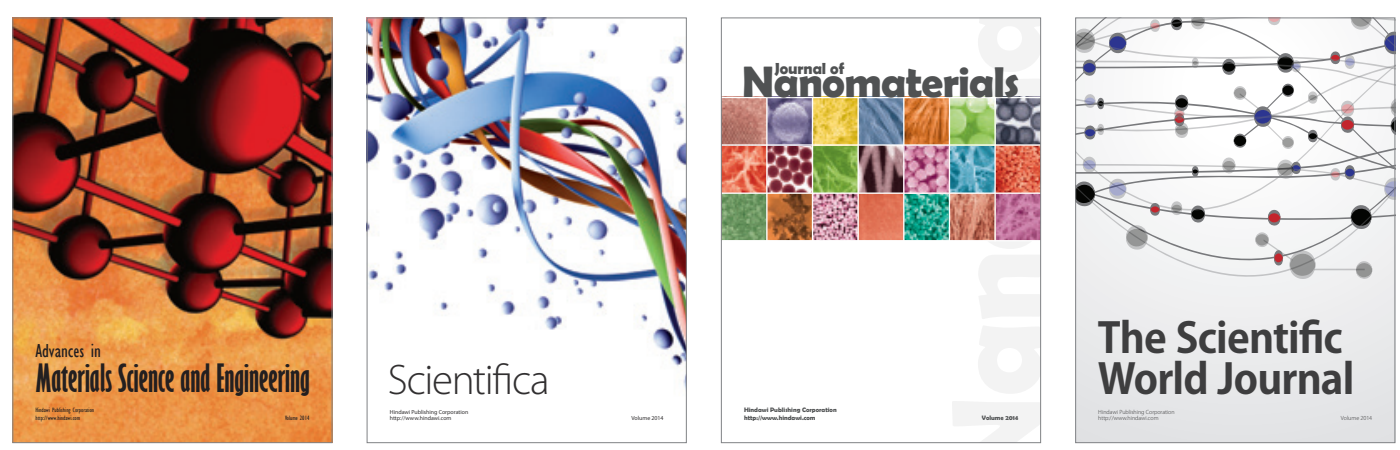

\section{The Scientific World Journal}
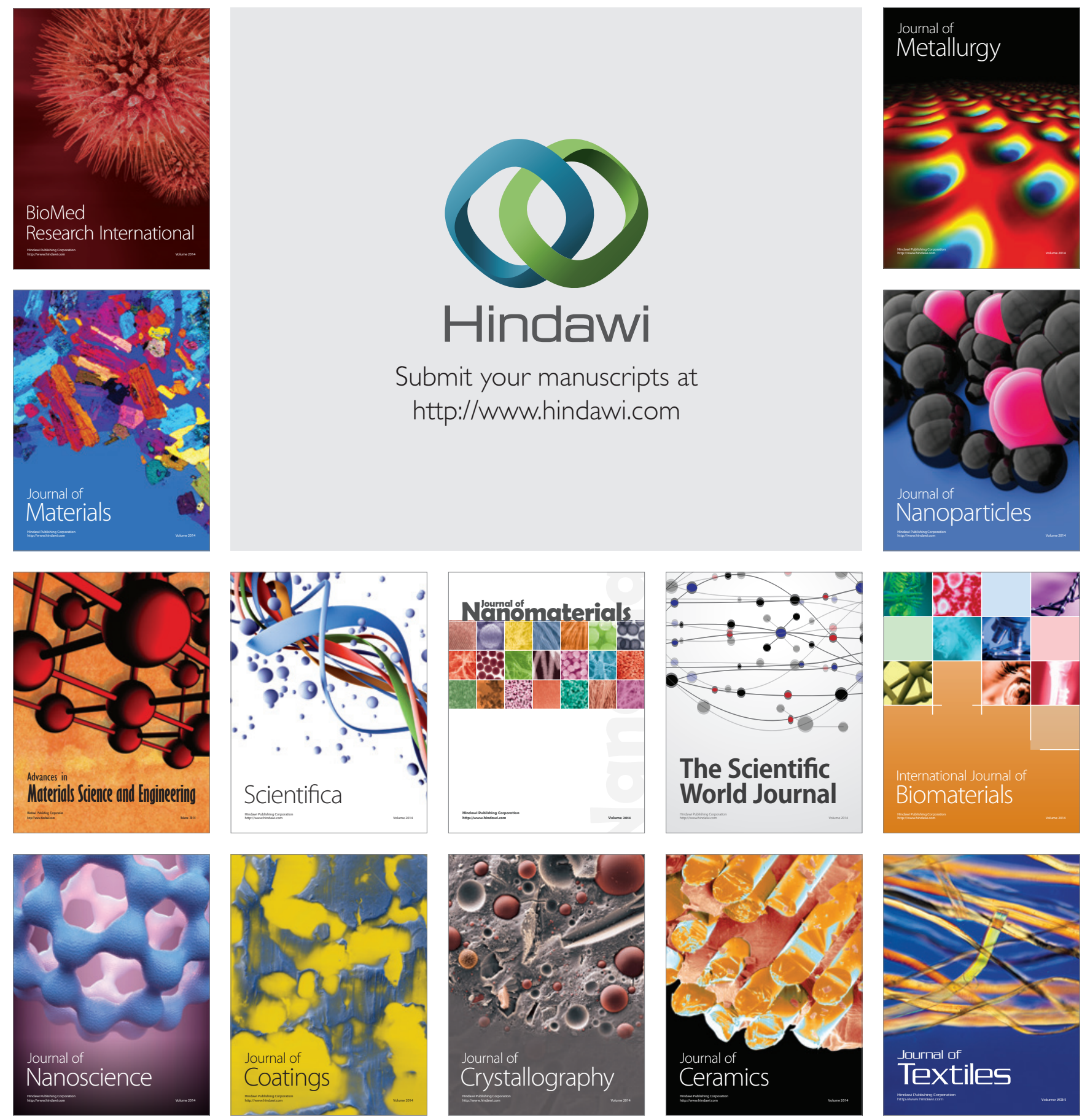